\title{
Invariant tori for a nonlinearly modified Kawahara equation with periodic boundary conditions
}

Li Yin ${ }^{1 *}$, Lufang Mi ${ }^{1}$, Wenyan Cui ${ }^{1}$ and Xiuli Lin²

"Correspondence: yinli_79@163.com; yinli7979@163.com

${ }^{1}$ College of Science, Binzhou University, Binzhou City, China Full list of author information is

available at the end of the article

\begin{abstract}
This paper is concerned with a nonlinearly modified Kawahara equation with periodic boundary conditions

$$
u_{t}+u_{x x x}-u_{5 x}+\alpha u^{2} u_{x}=0
$$

Based on an abstract infinite-dimensional KAM theorem for unbounded perturbation vector fields and partial Birkhoff normal form, we prove the existence of $n$-dimensional invariant tori for the equation.
\end{abstract}

MSC: $37 \mathrm{~K} 55 ; 35 \mathrm{Q} 55 ; 46 \mathrm{G05} ; 46 \mathrm{~N} 20$

Keywords: Nonlinearly modified Kawahara equation; KAM theorem; Invariant tori; Normal form

\section{Introduction and main result}

Let us begin with the nonlinear partial differential equation

$$
u_{t}+u_{x x x}-u_{5 x}+\alpha u^{2} u_{x}=0
$$

under periodic boundary conditions

$$
u(t, x+2 \pi)=u(t, x), \quad-\infty<t<\infty .
$$

Equation (1.1) is the famously modified Kawahara equation, arising in several physical applications, for example, in the theory of magneto-acoustic waves in plasma [17] and in the theory of long waves in shallow liquid under ice cover [27]. Demina and Kudryashov [14] studied meromorphic traveling wave solutions of the following partial differential equation:

$$
u_{t}+b u_{x x x}-c u_{5 x}+a u^{n} u_{x}=0
$$

Abbasbandy [1] obtained a family of traveling-wave solutions of the Kawahara equation by applying the homotopy analysis method (HAM). Some families of exact solutions to

(c) The Author(s) 2019. This article is distributed under the terms of the Creative Commons Attribution 4.0 International License (http://creativecommons.org/licenses/by/4.0/), which permits unrestricted use, distribution, and reproduction in any medium, provided you give appropriate credit to the original author(s) and the source, provide a link to the Creative Commons license, and indicate if changes were made. 
the Kawahara equation and the modified Kawahara equation also were given in [4, 31]. However, only a few papers discussed the existence of quasi-periodic solutions for the Kawahara equation by using KAM method.

It was well known that KAM of Hamilton partial differential equation originated from Kuksin and Wayne's work. We consider the Hamiltonian partial differential equation (HPDE)

$$
\dot{\omega}=A \omega+f(\omega)
$$

For some Sobolev space $\mathcal{H}^{p} \ni \omega$, the linear operator $A$ maps $\mathcal{H}^{p}$ into $\mathcal{H}^{p-\delta}$, and the nonlinear term $f$ sends some neighborhood of $\mathcal{H}^{p} \rightarrow \mathcal{H}^{p-a}$. The numbers $\delta \in \mathbb{R}^{+}$and $a$ are called the orders of $A$ and $f$, respectively.

When $a \leq 0$, the vector-field $f$ is called a bounded perturbation. Kuksin [18] and Wayne [34] proved the earliest KAM theorem for PDEs with bounded perturbation and applied it to the one-dimensional wave equation with Dirichlet bounded conditions

$$
u_{t t}=u_{x x}+M_{\xi} u+u^{3}=0, \quad u(t, 0)=u(t, \pi)=0,
$$

and the one-dimensional Schrödinger equation with Dirichlet boundary conditions

$$
i u_{t}=u_{x x}+M_{\xi} u+|u|^{2} u=0, \quad u(t, 0)=u(t, \pi)=0 .
$$

For these two equations, we have $a=-1, \delta=2$ and $a=0, \delta=2$, respectively. Unfortunately, when considering the one-dimensional wave and Schrödinger equations with periodic boundary conditions, the multiplicity of the eigenvalues becomes an obstacle. In the 1990s, the people even doubted that KAM could not handle multiple normal frequency case. To overcome this difficulty, using the Nash-Moser iteration and LyapunovSchmidt decomposition, Craig and Wayne $[10,11]$ gave a good estimation of the inverse of an infinite-dimensional matrix with small eigenvalues, and thus the quasi-periodic solutions were successfully constructed to the equations with periodic boundary conditions. Further developing Craig and Wayne's method, Bourgain [5-8] proved the existence of quasi-periodic solutions to higher-dimensional wave and Schrödinger equations. We need to emphasize that the methods of Craig, Wayne, and Bourgain are very effective methods to prove the existence of quasi-periodic solutions but do not get linear stability, so their results are weaker than those obtained by KAM method. On the other hand, You [35] established a KAM theorem for lower-dimensional tori in the finite-dimensional case and applied it to multiple normal frequency case. In 2000, Chierchia and You [9] proved an infinite-dimensional theorem that can deal with multiple normal frequency case. For more detail, we refer the readers to $[22,32,33,36,37]$ and the references therein.

When $a>0$, the vector-field $f$ is called an unbounded perturbation. For example, when the people think about $\mathrm{KdV}$ equation with periodic boundary conditions with $a=1, \delta=3$, new difficulties appear, such as homological equations with variable coefficients and a stronger inequality that the divisor need to satisfy to guarantee the boundedness of the transformation. Kuksin [20, 21] gave the first KAM theorem and applied it to KdV equation by overcoming this difficulty under the assumption $0<a<\delta-1$. See also [16]. Based on Kuksin's estimate of solutions of homological equations with nonconstant coefficients 
[19], the existence of a family of linearly stable quasi-periodic solutions for the KdV equation with periodic boundary conditions was presented. Very recently, Cui et al. [13] were concerned with small perturbation of the nonlinear partial differential equation

$$
u_{t}=u_{5 x}-\frac{5}{16}\left(8 u_{x x}^{2}+8 u_{x} u_{x x x}\right)
$$

under periodic boundary conditions. They obtained the existence of many two-dimensional invariant tori for this equation by using an infinite-dimensional KAM theorem.

When $0<a=\delta-1$, the vector-field $f$ is called the limiting case. In 2010, by careful analysis of a Töplitz matrix and its exponential Liu and Yuan [24] established a new estimate for the small-denominator equation with critical unbounded variable coefficients. Hence, a KAM theorem for infinite-dimensional Hamiltonian systems with $0<a=\delta-1$ was given in [25]. More results on PDEs with unbounded perturbation, see [12, 23, 26, 28-30] and the references therein.

When $a=\delta$, Baldi, Berti, and Montalto [3] proved the existence of quasi-periodic solutions for the perturbed Airy equation

$$
u_{t}+u_{x x x}+\varepsilon \partial_{x}\left(f\left(\lambda t, x, u, u_{x}, u_{x x}\right)\right)=0, \quad x \in \mathbb{T}, f \in \mathbf{C}^{k},
$$

where $\delta=3$ and $a=3$. The crucial point of this problem is the reduction of the linear Airy equation. We refer the reader to $[2,15]$.

The research in this area is very active at present, and the people are developing the KAM method to deal with more complex Hamilton partial differential equations. In this paper, the modified Kawahara equation with periodic boundary conditions belongs to uncritical unbounded perturbation case with $a=1$ and $\delta=5$. Hence the existence of $n$ dimensional invariant tori was proven by using Kuksin's KAM theorem in [16].

Now we study (1.1) as an infinite-dimensional Hamiltonian system on some suitable phase space. For any integer $p>0$, the phase space

$$
\mathcal{H}^{p}=\left\{u \in L^{2}(S ; \mathbb{R}):\|u\|_{p}<\infty\right\}
$$

of the real-valued functions on $S=\mathbb{R} / 2 \pi \mathbb{Z}$, where

$$
\|u\|_{p}^{2}=|\hat{u}(0)|^{2}+\sum_{k \in \mathbb{Z}}|k|^{2 p}|\hat{u}(k)|^{2}
$$

is defined in terms of the Fourier transform $\hat{u}$ of $u, u(x)=\sum_{k \in \mathbb{Z}} \hat{u}(k) e^{\mathrm{i} k x}$. We endow $\mathcal{H}^{p}$ with the Poisson structure proposed by Gardner:

$$
\{F, G\}=\int_{S} \frac{\partial F}{\partial u(x)} \frac{\mathrm{d}}{\mathrm{d} x} \frac{\partial G}{\partial u(x)} \mathrm{d} x
$$

where $F, G$ are differential functions on $\mathcal{H}^{p}$ with $L^{2}$-gradients in $\mathcal{H}^{1}$. Under the standard inner product on $L^{2}(S)$, the modified Kawahara equation (1.1) can be written in the form

$$
u_{t}=\frac{\mathrm{d}}{\mathrm{d} x}\left(\frac{\partial H}{\partial u}\right)
$$


with Hamiltonian

$$
H(u)=\int_{S}\left(\frac{1}{2} u_{x x}^{2}+\frac{1}{2} u_{x}^{2}-\frac{\alpha}{12} u^{4}\right) \mathrm{d} x .
$$

Now consider the phase space

$$
\mathcal{H}_{0}^{p}=\left\{u \in \mathcal{H}^{p}:[u]=0\right\}
$$

where $[u]=\int_{S} u(x) \mathrm{d} x$. The Poisson structure on $\mathcal{H}_{0}^{p}$ is the same as that on $\mathcal{H}^{p}$. In fact, set $u=v+c$, where $[v]=0$ and $[u]=c$. Then the Hamiltonian of the modified Kawahara equation is

$$
H_{c}(u)=\int_{S}\left(\frac{1}{2} v_{x x}^{2}+\frac{1}{2} v_{x}^{2}-\frac{\alpha}{12} v^{4}\right) \mathrm{d} x-\int_{S}\left(\frac{\alpha}{3} c v^{3}\right) \mathrm{d} x-\int_{S}\left(\frac{\alpha}{2} c^{2} v^{2}\right) \mathrm{d} x-\frac{\pi \alpha}{6} c^{4},
$$

where $c$ is considered as a real parameter. For simplicity, we only consider $c=0$ in the following sections.

Our main result reads as follows.

Theorem 1.1 Consider the nonlinearly modified Kawahara equation

$$
\frac{\partial u}{\partial t}=-u_{x x x}+u_{5 x}-\alpha u^{2} u_{x}
$$

where the Hamiltonian function $H$ is defined by (1.5). Then, for any given index set $\mathcal{J}=$ $\left\{j_{1}<j_{2}<\cdots<j_{n}\right\} \subset \mathbb{N}$, there exists $\varepsilon_{0}>0$, depending only on $\mathcal{J}$, such that, for $0<\varepsilon<\varepsilon_{0}$, there exist

(1) a nonempty Cantor set $\Pi_{\varepsilon} \subset \Pi$ with meas $\left(\Pi \backslash \Pi_{\varepsilon}\right) \rightarrow 0$ as $\varepsilon \rightarrow 0$, where $\Pi$ is a compact subset of $\mathbb{R}^{n}$ of positive Lebesgue measure,

(2) a Lipschitz family of real analytic torus embeddings

$$
\Phi: \mathbb{T}^{n} \times \Pi_{\varepsilon} \rightarrow S_{p}^{2}
$$

where $S_{p}^{2}=\mathbb{T}^{n} \times \mathbb{R}^{n} \times \ell^{p} \times \ell^{p}$, and $\ell^{p}$ is the Hilbert space of all complex-valued sequences with norm (2.2).

(3) a Lipschitz map $\phi: \Pi_{\varepsilon} \rightarrow \mathbb{R}^{n}$ such that, for each $(\theta, \xi) \in \mathbb{T}^{n} \times \Pi_{\varepsilon}$, the curve $u(t)=\Phi(\theta+\phi(\xi) t, \xi)$ is a quasi-periodic solution of equation (1.6) winding around the invariant $\Phi\left(\mathbb{T}^{n} \times\{\xi\}\right)$. Moreover, each such torus is linearly stable.

\section{Proof of the main theorem}

First of all, we change the nonlinearly modified Kawahara equation (1.1) into Hamiltonian form of infinitely many coordinates and then transform it into a partial normal form up to order four. In the second subsection, we give the proof of Theorem 1.1.

\subsection{Normal form}

We write

$$
u(t, x)=\sum_{j \neq 0} \gamma_{j} q_{j}(t) e_{j}(x)
$$


where $\gamma_{j}=\sqrt{|j|}, e_{j}(x)=\frac{1}{\sqrt{2 \pi}} e^{\mathrm{i} j x}$. The coordinates are taken from the Hilbert space $\ell^{p}$ of all complex-valued sequences $\left(q_{j}\right)_{j \neq 0}$ with

$$
\|q\|_{p}^{2}=\sum_{j \neq 0}|j|^{2 p}\left|q_{j}\right|^{2}<\infty, \quad q_{-j}=\bar{q}_{j}
$$

Now Hamiltonian (1.5) can be written as

$$
\dot{q}_{j}=\mathrm{i} \sigma_{j}\left(\frac{\partial H}{\partial q_{-j}}\right), \quad \sigma_{j}= \begin{cases}1, & j \geq 1, \\ -1, & j \leq-1,\end{cases}
$$

with the new Hamiltonian

$$
H(q)=\sum_{j \geq 1} \lambda_{j}\left|q_{j}\right|^{2}-\frac{\alpha}{24 \pi} \sum_{k+l+m+n=0} \gamma_{k} \gamma_{l} \gamma_{m} \gamma_{n} q_{k} q_{l} q_{m} q_{n}=\Lambda+G
$$

where $\lambda_{j}=j^{5}+j^{3}$, and the corresponding symplectic structure is

$$
-\mathrm{i} \sum_{j \geq 1} d q_{j} \wedge d q_{-j}
$$

So, the associated Hamilton vector field with Hamiltonian $H$ is given by

$$
X_{H}=\mathrm{i} \sigma_{j} \sum_{j \neq 0} \frac{\partial H}{\partial q_{-j}} \frac{\partial}{\partial q_{j}} .
$$

Lemma 2.1 The Hamiltonian vector field $X_{G}$ is real analytic as a map from $\ell^{p}$ into $\ell^{p-1}$ for each $p>\frac{3}{2}$. Moreover, $\left\|X_{G}\right\|_{p-1}=O\left(\|q\|_{p}^{3}\right)$.

Proof Since

$$
G(q)=-\frac{\alpha}{24 \pi} \sum_{k+l+m+n=0} \gamma_{k} \gamma_{l} \gamma_{m} \gamma_{n} q_{k} q_{l} q_{m} q_{n},
$$

we easily obtain

$$
\left|\frac{\partial G}{\partial q_{-j}}\right| \leq \frac{\alpha}{6 \pi} \sum_{j=k+l+m} \gamma_{-j} \gamma_{k} \gamma_{l} \gamma_{m}\left|q_{k} q_{l} q_{m}\right|=\frac{\alpha}{6 \pi} \gamma_{j} g_{j},
$$

where $g_{j}=\sum_{j=k+l+m} \gamma_{k} \gamma_{l} \gamma_{m}\left|q_{k} q_{l} q_{m}\right|$.

Defining $\omega=\left(\omega_{j}\right)_{j}=\left(\gamma_{j}\left|q_{j}\right|\right)_{j}, g=\left(g_{j}\right)$, we get $g_{j}=(\omega * \omega * \omega)_{j}$, so $g=\omega * \omega * \omega$. For $q \in \ell^{p}$, it is obvious that $\omega \in \ell^{p-\frac{1}{2}}$. Hence we have

$$
\|g\|_{p-\frac{1}{2}}=\|\omega * \omega * \omega\|_{p-\frac{1}{2}} \leq C\|\omega\|_{p-\frac{1}{2}}^{3} \leq C\|q\|_{p}^{3},
$$

and consequently

$$
\left\|\partial_{q} G\right\|_{p-1} \leq C\|g\|_{p-\frac{1}{2}} \leq C\|q\|_{p}^{3} .
$$


Lemma 2.2 Suppose $k, l, m, n \in \mathbb{Z} \backslash\{0\}$ and denote

$$
\begin{aligned}
& \Delta=\left\{(k, l, m, n) \in \mathbb{Z}^{4} \backslash\{0\} \mid k+l+m+n=0\right\}, \\
& \Delta_{1}=\{(k, l, m, n) \in \Delta \mid k+l, k+m, k+n \neq 0\} .
\end{aligned}
$$

Then we have

$$
\left|\lambda_{k}+\lambda_{l}+\lambda_{m}+\lambda_{n}\right| \geq \frac{5}{4} \max \left\{|k|^{3},|l|^{3},|m|^{3},|n|^{3}\right\}
$$

for $(k, l, m, n) \in \Delta_{1}$.

Proof As $(k, l, m, n) \in \Delta_{1}$, we easily obtain

$$
\begin{aligned}
k^{5}+l^{5}+m^{5}+n^{5} & =k^{5}+l^{5}+m^{5}-(k+l+m)^{5} \\
& =-5(k+l)(k+m)(k+n)\left(k^{2}+l^{2}+m^{2}+k m+k l+l m\right) \\
& =\frac{5}{2}(k+l)(k+m)(k+n)\left(k^{2}+l^{2}+m^{2}+n^{2}\right)
\end{aligned}
$$

and

$$
k^{3}+l^{3}+m^{3}+n^{3}=3(k+l)(k+m)(k+n) .
$$

On the other hand, we know from [12] that

$$
|(k+l)(k+m)(k+n)| \geq \frac{1}{2} \max \{|k|,|l|,|m|,|n|\}
$$

and hence

$$
\begin{aligned}
\left|\lambda_{k}+\lambda_{l}+\lambda_{m}+\lambda_{n}\right| & =|(k+l)(k+m)(k+n)|\left|\frac{5}{2}\left(k^{2}+l^{2}+m^{2}+n^{2}\right)+3\right| \\
& >|(k+l)(k+m)(k+n)|\left|\frac{5}{2}\left(k^{2}+l^{2}+m^{2}+n^{2}\right)\right| \\
& =\frac{5}{4} \max \left\{|k|^{3},|l|^{3},|m|^{3},|n|^{3}\right\} .
\end{aligned}
$$

Lemma 2.3 There exists a real analytic symplectic coordinate transformation $\Phi$ defined in a neighborhood of the origin of $\ell^{p}$ that transforms the Hamiltonian (2.4) into its normal form up to order four. That is,

$$
H_{1}=H \circ \Phi=\Lambda-B+\tilde{P}
$$

with

$$
\begin{aligned}
& B=\frac{\alpha}{24 \pi}\left(3 \sum_{k \neq l}|k||l|\left|q_{k}\right|^{2}\left|q_{l}\right|^{2}+2 \sum_{k \neq 0}|k|^{2}\left|q_{k}\right|^{4}\right), \\
& \left\|\bar{P}_{q}\right\|_{p-1}=O\left(\|q\|_{p}^{5}\right) .
\end{aligned}
$$


Proof Let us begin to normalize the fourth-order term $G$ of $q$. Let the transformation $\Phi_{1}=X_{F}^{1}$ be the time-1-map of the flow of the Hamiltonian vector field $X_{F}$. Then

$$
\begin{aligned}
H_{1} & =H \circ \Phi_{1}=H \circ X_{F}^{1} \\
& =\Lambda+\{\Lambda, F\}+G+\int_{0}^{1}(1-t)\{\{\Lambda, F\}, F\} \circ X_{F}^{t} d t+\int_{0}^{1}\{G, F\} \circ X_{F}^{t} d t \\
& =\Lambda+\{\Lambda, F\}+G+\bar{P} \\
& =\Lambda+\{\Lambda, F\}-B+G_{1}+\bar{P},
\end{aligned}
$$

where

$$
\bar{P}=\int_{0}^{1}(1-t)\{\{\Lambda, F\}, F\} \circ X_{F}^{t} d t+\int_{0}^{1}\{G, F\} \circ X_{F}^{t} d t
$$

and $G_{1}=G+B$.

To solve the equation $\{\Lambda, F\}+G_{1}=0$, we define

$$
F=\sum_{k, l, m, n \neq 0} F_{k l m n} q_{k} q_{l} q_{m} q_{n}
$$

by

$$
-\mathrm{i} F_{k l m n}= \begin{cases}\frac{\alpha \gamma_{k} \gamma_{l} \gamma_{m} \gamma_{n}}{24 \pi\left(\lambda_{k}+\lambda_{l}+\lambda_{m}+\lambda_{n}\right)}, & (k, l, m, n) \in \Delta_{1} \\ 0 & \text { otherwise }\end{cases}
$$

Then the Hamiltonian changes to

$$
H_{1}=\Lambda-B+\bar{P}
$$

Obviously, the function $\bar{P}$ is real analytic in $\ell^{p}$ with real value, and its gradient is analytic $\ell^{p}$ into $\ell^{p-1}$ with $\left\|\bar{P}_{q}\right\|_{p-1}=O\left(\|q\|_{p}^{5}\right)$.

In the next step, we need to establish the regularity of the vector field $X_{F}$. Since the $j$ th element of the gradient $\partial_{q} F$ reads explicitly,

$$
\frac{\partial F}{\partial q_{-j}}=\sum_{l+m+n=j}\left(F_{(-j) l m n}+F_{l(-j) m n}+F_{l m(-j) n}+F_{l m n(-j)}\right) q_{l} q_{m} q_{n} .
$$

Thus we get the estimate

$$
\left|\frac{\partial F}{\partial q_{-j}}\right| \leq \frac{2 \alpha}{15 \pi \gamma_{j}^{5}} \sum_{l+m+n=j} \gamma_{l} \gamma_{m} \gamma_{n}\left|q_{l}\right|\left|q_{m}\right|\left|q_{n}\right|:=\frac{2 \alpha}{15 \pi \gamma_{j}^{5}} r_{j}
$$

where

$$
r_{j}=\sum_{l+m+n=j} \gamma_{l} \gamma_{m} \gamma_{n}\left|q_{l}\right|\left|q_{m}\right|\left|q_{n}\right|
$$


Then

$$
\left\|\partial_{q} F\right\|_{p+2}=O\left(\|q\|_{p}^{3}\right)
$$

and the proof of the lemma is completed.

\subsection{Proof of Theorem 1.1}

For any given index set $\mathcal{J}$, considering the transformation $\Phi$ in Lemma 2.3, we get the new Hamiltonian

$$
H_{1}=\Lambda-B+\bar{P}
$$

which is analytic in some neighborhood of the origin of $\ell^{p}$.

We introduce new symplectic polar and real coordinates $(\varphi, y, z, \bar{z})$ by setting

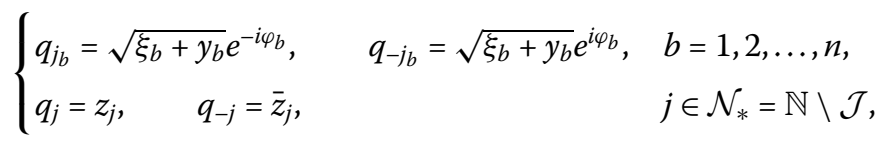

where $\xi=\left(\xi_{1}, \xi_{2}, \ldots, \xi_{n}\right) \in \mathbb{R}^{n}$. Then

$$
\begin{aligned}
\Lambda= & \sum_{1 \leq b \leq n} \lambda_{j_{b}}\left(\xi_{b}+y_{b}\right)+\sum_{j \in \mathcal{N}_{*}} \lambda_{j} z_{j} \bar{z}_{j}, \\
B= & \frac{\alpha}{24 \pi}\left(3 \sum_{k \neq l}|k||l|\left|q_{k}\right|^{2}\left|q_{l}\right|^{2}+2 \sum_{k \neq 0}|k|^{2}\left|q_{k}\right|^{4}\right) \\
= & \frac{\alpha}{24 \pi}\left(12 \sum_{k, l>0} k l\left|q_{k}\right|^{2}\left|q_{l}\right|^{2}+4 \sum_{k>0}|k|^{2}\left|q_{k}\right|^{4}\right) \\
= & \frac{\alpha}{24 \pi}\left[12 \sum_{1 \leq a \neq b \leq n} j_{a} j_{b}\left(\xi_{a}+y_{a}\right)\left(\xi_{b}+y_{b}\right)\right] \\
& +\frac{\alpha}{24 \pi}\left[24 \sum_{\substack{1 \leq b \leq n \\
j \in \mathcal{N}_{*}}} j j_{b}\left(\xi_{b}+y_{b}\right) z_{j} \bar{z}_{j}\right] \\
& +\frac{\alpha}{24 \pi}\left[12 \sum_{\substack{j, j^{\prime} \in \mathcal{N}_{*} \\
j \neq j^{\prime}}} j i j^{\prime} z_{j} \bar{z}_{j} z_{j^{\prime}} z_{j^{\prime}}\right] \\
& +\frac{\alpha}{24 \pi}\left[4 \sum_{\substack{1 \leq b \leq n \\
1 \leq b \leq}}\left(j_{b}\right)^{2}\left(\xi_{b}+y_{b}\right)^{2}+4 \sum_{j \in \mathcal{N}_{*}} j^{2} z_{j}^{2} \bar{z}_{j}^{2}\right] .
\end{aligned}
$$

Thus the new Hamiltonian, denoted by $H_{2}$, up to a constant depending on $\xi$, is given by

$$
H_{2}=N+P=\sum_{1 \leq b \leq n} \omega_{b} y_{b}+\sum_{j \in \mathcal{N}_{*}} \Omega_{j} z_{j} \bar{z}_{j}-Q+\bar{P}
$$

with symplectic structure

$$
\sum_{1 \leq b \leq n} d \varphi_{b} \wedge d y_{b}-\mathrm{i} \sum_{j \in \mathcal{N}_{*}} d z_{j} \wedge d \bar{z}_{j}
$$


where

$$
\begin{aligned}
& \omega_{b}=\lambda_{j_{b}}-\sum_{a \neq b}\left(\frac{\alpha}{\pi} j_{a j} j_{b} \xi_{a}-\frac{\alpha}{3 \pi} j_{b}^{2} \xi_{b}\right), \\
& \Omega_{j}=\lambda_{j}-\frac{\alpha}{\pi} \sum_{1 \leq b \leq n} j j_{b} \xi_{b},
\end{aligned}
$$

and $P=-Q+\bar{P}$ with

$$
\begin{aligned}
Q= & \frac{\alpha}{24 \pi}\left(\sum_{a \neq b} 12 j_{a} j_{b} y_{a} y_{b}+24 \sum_{\substack{1 \leq b \leq n \\
j \in \mathcal{N}_{*}}} j j_{b} y_{b} z_{j} \bar{z}_{j}\right) \\
& +\frac{\alpha}{24 \pi}\left(12 \sum_{\substack{j, j^{\prime} \in \mathcal{N}_{*} \\
j \neq j^{\prime}}} j j^{\prime} z_{j} \bar{z}_{j} z_{j^{\prime}} \overline{z^{\prime}}+4 \sum_{1 \leq b \leq n} j_{b}^{2} y_{b}^{2}+4 \sum_{j \in \mathcal{N}_{*}} j^{2} z_{j}^{2} \bar{z}_{j}^{2}\right) .
\end{aligned}
$$

Now consider the phase space domain

$$
\left\{D(s, r):|\Im \varphi|<s,|y|<r^{2},\|u\|_{p}+\|v\|_{p}<r\right\},
$$

where for the definition of norm $\|u\|_{p}$ and more notations, we refer to the Appendix. Next, we will check Assumptions A, B, and C of the KAM Theorem A.1 in the Appendix.

Regarding $\Omega$ as an infinite-dimensional column vector with its index $j \in \mathcal{N}_{*}$, from (2.8), we have

$$
\Omega_{j}(\xi)=\bar{\Omega}_{j}+\tilde{\Omega}_{j}(\xi)
$$

where $\bar{\Omega}_{j}=\lambda_{j}=j^{5}+j^{3}$ is independent of $\xi$. Furthermore, we also get

$$
\left|\Omega_{j}\right|_{\Pi}^{\operatorname{lip}} \leq \frac{|\alpha|}{\pi} \sum_{\substack{1 \leq b \leq n, j \in \mathcal{N}_{*}}}\left|j_{b}\right||j| \leq \frac{|\alpha|}{\pi} \max _{1 \leq b \leq n}\left\{\left|j_{b}\right||j|\right\} .
$$

Thus

$$
|\Omega|_{-1, \Pi}^{\text {lip }}=\sup _{j \in \mathcal{N}_{*}} j^{-1}\left|\Omega_{j}\right|_{\Pi}^{\operatorname{lip}} \leq \frac{|\alpha|}{\pi} \max _{1 \leq b \leq n}\left\{\left|j_{b}\right|\right\}:=M_{1}
$$

which means that Assumption A is fulfilled with $d=5$ and $\delta=1$. Therefore the functions

$$
\xi \mapsto \frac{\tilde{\Omega}_{j}(\xi)}{j}, \quad j \geq 1,
$$

are uniformly Lipschitz on $\Pi_{\varepsilon}$,

In view of (2.7), we have that $\xi \mapsto \omega$ is an affine transformation from $\Pi_{\varepsilon}$ to $\mathbb{R}^{n}$. Consider

$$
\omega=\breve{\omega}-\frac{\alpha}{3 \pi} A \xi
$$


where

$$
\begin{aligned}
\breve{\omega} & =\left(\begin{array}{c}
\lambda_{j_{1}} \\
\lambda_{j_{2}} \\
\cdots \\
\lambda_{j_{n}}
\end{array}\right)=\left(\begin{array}{c}
j_{1}^{5}+j_{1}^{3} \\
j_{2}^{5}+j_{2}^{3} \\
\ldots \\
j_{n}^{5}+j_{n}^{3}
\end{array}\right), \quad \xi=\left(\begin{array}{c}
\xi_{1} \\
\xi_{2} \\
\ldots \\
\xi_{n}
\end{array}\right), \\
A & =\left(\begin{array}{ccccc}
j_{1}^{2} & 3 j_{1} j_{2} & 3 j_{1} j_{3} & \cdots & 3 j_{1} j_{n} \\
3 j_{2} j_{1} & j_{2}^{2} & 3 j_{2} j_{3} & \cdots & 3 j_{2} j_{n} \\
3 j_{3} j_{1} & 3 j_{3} j_{2} & j_{3}^{2} & \cdots & 3 j_{3} j_{n} \\
\cdots & \cdots & \cdots & \cdots & \cdots \\
3 j_{n} j_{1} & 3 j_{n} j_{2} & 3 j_{n} j_{3} & \cdots & j_{n}^{2}
\end{array}\right) .
\end{aligned}
$$

Hence simple computation yields

$$
\operatorname{det} A=(-2)^{n-1}(3 n-2) j_{1}^{2} j_{2}^{2} \cdots j_{n}^{2} \neq 0,
$$

and therefore the real map $\xi \mapsto \omega$ is a lipeomorphism between $\Pi$ and its image. This implies that the first part of Assumption B is fulfilled with positive $M_{2}$ and $L$ depending only on the set $\mathcal{J}$.

We further check the second part of Assumption B. Rewriting

$$
\Omega(\xi)=\bar{\Omega}-\frac{\alpha}{\pi} B \xi,
$$

where $\bar{\Omega}$ is an infinite-dimensional column vector with its $j$ th element $\bar{\Omega}_{j}=j^{5}+j^{3}$, and $B$ is a $-\infty \times n$ matrix with its $j$ th row $B_{j}=j\left(j_{1}, j_{2}, \ldots, j_{n}\right)$. We have to check that, for all $k=\left(k_{1}, k_{2}, \ldots, k_{n}\right) \in \mathbb{Z}^{n}$ and $1 \leq|l| \leq 2$ with $l \in \mathbb{Z}^{\infty}$,

$$
\operatorname{meas}\left\{\xi \in \Pi_{\varepsilon}:\langle k, \omega(\xi)\rangle+\langle l, \Omega(\xi)\rangle=0\right\}=0 .
$$

Take

$$
\begin{aligned}
\mathfrak{g}(\xi) & =\langle k, \omega(\xi)\rangle+\langle l, \Omega(\xi)\rangle \\
& =\langle k, \breve{\omega}\rangle+\langle l, \bar{\Omega}\rangle+\left\langle k,-\frac{\alpha}{3 \pi} A \xi\right\rangle+\left\langle l,-\frac{\alpha}{\pi} B \xi\right\rangle .
\end{aligned}
$$

From condition (2.9) it follows that

$$
\langle k, \breve{\omega}\rangle+\langle l, \bar{\Omega}\rangle \neq 0 \quad \text { or } \quad k A+3 l B \neq 0 .
$$

Indeed, if we suppose that

$$
k A+3 l B=0,
$$

then

$$
k=-3 l B A^{-1}=-l \hat{B},
$$


where $\hat{B}=3 B A^{-1}=\left(\hat{B}_{j}\right)$. Direct calculation results in

$$
A^{-1}=\frac{1}{6 n-4}\left(\begin{array}{ccccc}
\frac{5-3 n}{j_{1}^{2}} & \frac{3}{j_{1} j_{2}} & \frac{3}{j_{1} j_{3}} & \cdots & \frac{3}{j_{1} j_{n}} \\
\frac{3}{j_{2} j_{1}} & \frac{5-3 n}{j_{2}^{2}} & \frac{3}{j_{2} j_{3}} & \cdots & \frac{3}{j_{2} j_{n}} \\
\frac{3}{j_{3} j_{1}} & \frac{3}{j_{3} j_{2}} & \frac{5-3 n}{j_{3}^{2}} & \cdots & \frac{3}{j_{3 j} j_{n}} \\
\cdots & \cdots & \cdots & \cdots & \cdots \\
\frac{3}{j_{n} j_{1}} & \frac{3}{j_{n} j_{2}} & \frac{3}{j_{n} j_{3}} & \cdots & \frac{5-3 n}{j_{n}^{2}}
\end{array}\right) .
$$

So, we easily obtain

$$
\hat{B}_{j}=\frac{j}{3 n-2}\left(\frac{1}{j_{1}}, \frac{1}{j_{2}}, \ldots, \frac{1}{j_{n}}\right) .
$$

We easily see that $k A+3 l B \neq 0$, except for the following four cases:

(1) $l_{j}= \begin{cases} \pm 1, & j=\frac{h(3 n-2)}{3}\left[j_{1}, j_{2}, \ldots, j_{n}\right] \\ 0 & \text { otherwise }\end{cases}$

$k=\mp\left[j_{1}, j_{2}, \ldots, j_{n}\right]\left(\frac{1}{j_{1}}, \frac{1}{j_{2}}, \ldots, \frac{1}{j_{n}}\right), \quad h \in \mathbb{N}$,

(2) $l_{j}= \begin{cases} \pm 2, & j=\frac{h(3 n-2)}{6}\left[j_{1}, j_{2}, \ldots, j_{n}\right] \\ 0 & \text { otherwise }\end{cases}$

$k=\mp h\left[j_{1}, j_{2} \cdots j_{n}\right]\left(\frac{1}{j_{1}}, \frac{1}{j_{2}} \cdots \frac{1}{j_{n}}\right), \quad h \in \mathbb{N}$,

(3) $l_{j}= \begin{cases} \pm 1, & j=j^{\prime}, j^{\prime \prime}, 3\left(j^{\prime}+j^{\prime \prime}\right)=h(3 n-2)\left[j_{1}, j_{2}, \ldots, j_{n}\right], \\ 0 & \text { otherwise, }\end{cases}$

$k=\mp \frac{3\left(j^{\prime}+j^{\prime \prime}\right)}{3 n-2}\left(\frac{1}{j_{1}}, \frac{1}{j_{2}}, \ldots, \frac{1}{j_{n}}\right), \quad h \in \mathbb{N}$,

(4) $l_{j}= \begin{cases} \pm 1, & j=j^{\prime}, \\ \pm 1, & j=j^{\prime \prime}<j^{\prime} \text { and } 3\left(j^{\prime}-j^{\prime \prime}\right)=h(3 n-2)\left[j_{1}, j_{2}, \ldots, j_{n}\right], \\ 0 & \text { otherwise, }\end{cases}$

$$
k=\mp \frac{3\left(j^{\prime}-j^{\prime \prime}\right)}{3 n-2}\left(\frac{1}{j_{1}}, \frac{1}{j_{2}}, \ldots, \frac{1}{j_{n}}\right), \quad h \in \mathbb{N},
$$

where $\left[j_{1}, j_{2}, \ldots, j_{n}\right]$ is the least common multiple of $j_{1}, j_{2}, \ldots, j_{n}$ Next, we check that

$$
\langle k, \breve{\omega}\rangle+\langle l, \bar{\Omega}\rangle \neq 0
$$

due to these four cases. Therefore we only check (2.12) for cases (1) and (3) under the proper condition. The others may be obtained by using the same method. 
Case 1. If (1) holds, then we have

$$
\begin{aligned}
\langle k, \breve{\omega}\rangle+\langle l, \bar{\Omega}\rangle= & \mp h\left[j_{1}, j_{2}, \ldots, j_{n}\right]\left(\sum_{i=1}^{n} j_{i}^{2}+\sum_{i=1}^{n} j_{i}^{4}\right) \\
& \pm\left(\frac{h(3 n-2)}{3}\left[j_{1}, j_{2}, \ldots, j_{n}\right]\right)^{5} \pm\left(\frac{h(3 n-2)}{3}\left[j_{1}, j_{2}, \ldots, j_{n}\right]\right)^{3} \\
= & \mp h\left[j_{1}, j_{2}, \ldots, j_{n}\right]\left(\sum_{i=1}^{n} j_{i}^{2}+\sum_{i=1}^{n} j_{i}^{4}-h^{4}\left(\frac{(3 n-2)}{3}\right)^{5}\left(\left[j_{1}, j_{2}, \ldots, j_{n}\right]\right)^{4}\right) \\
& \mp h\left[j_{1}, j_{2}, \ldots, j_{n}\right]\left(-h^{2}\left(\frac{(3 n-2)}{3}\right)^{3}\left(\left[j_{1}, j_{2}, \ldots, j_{n}\right]\right)^{2}\right) .
\end{aligned}
$$

If $\left[j_{1}, j_{2}, \ldots, j_{n}\right] \neq j_{n}$, then $\left[j_{1}, j_{2}, \ldots, j_{n}\right] \geq 2 j_{n}$. Hence we have

$$
\begin{aligned}
& \sum_{i=1}^{n} j_{i}^{2}<n j_{n}^{2} \leq \frac{n}{4}\left(\left[j_{1}, j_{2}, \ldots, j_{n}\right]\right)^{2}<h^{2}\left(\frac{(3 n-2)}{3}\right)^{3}\left(\left[j_{1}, j_{2}, \ldots, j_{n}\right]\right)^{3}, \\
& \sum_{i=1}^{n} j_{i}^{4}<n j_{n}^{4} \leq \frac{n}{16}\left(\left[j_{1}, j_{2}, \ldots, j_{n}\right]\right)^{4}<h^{4}\left(\frac{(3 n-2)}{3}\right)^{5}\left(\left[j_{1}, j_{2}, \ldots, j_{n}\right]\right)^{4} .
\end{aligned}
$$

This implies formula (2.12).

In case (3), we have

$$
\begin{aligned}
& \langle k, \breve{\omega}\rangle+\langle l, \bar{\Omega}\rangle \\
& =\mp\left(j^{\prime}+j^{\prime \prime}\right)\left[\frac{3}{3 n-2}\left(\sum_{i=1}^{n} j_{i}^{4}+\sum_{i=1}^{n} j_{i}^{2}\right)-\frac{\left(j^{\prime}\right)^{5}+\left(j^{\prime \prime}\right)^{5}+\left(j^{\prime}\right)^{3}+\left(j^{\prime \prime}\right)^{3}}{j^{\prime}+j^{\prime \prime}}\right] .
\end{aligned}
$$

When $n \geq 2$, we easily obtain

$$
\begin{aligned}
\sqrt{\frac{3}{3 n-2} \sum_{i=1}^{n} j_{i}^{2}} & <\sqrt{\frac{3 n\left(j_{n}\right)^{2}}{3 n-2}}<\frac{4}{3} j_{n} \leq \frac{4}{6}\left[j_{1}, j_{2}, \ldots, j_{n}\right] \\
& \leq \frac{3 n-2}{6}\left[j_{1}, j_{2}, \ldots, j_{n}\right] \leq \frac{j^{\prime}+j^{\prime \prime}}{2}<\sqrt{\frac{\left(j^{\prime}\right)^{3}+\left(j^{\prime \prime}\right)^{3}}{j^{\prime}+j^{\prime \prime}}}
\end{aligned}
$$

Completely similarly, we have

$$
\sqrt[4]{\frac{3}{3 n-2} \sum_{i=1}^{n} j_{i}^{4}}<\frac{j^{\prime}+j^{\prime \prime}}{2}<\sqrt[4]{\frac{\left(j^{\prime}\right)^{5}+\left(j^{\prime \prime}\right)^{5}}{j^{\prime}+j^{\prime \prime}}} .
$$

So the proof of case (3) is complete.

It remains to check Assumption C. It is easy to see that the Hamiltonian vector field of the perturbation $P=Q+\bar{P}+\varepsilon K \circ \Phi$ defines a map

$$
X_{P}: D(s, r) \times \Pi \rightarrow \mathfrak{S}_{p-2, \mathbb{C}}^{2}
$$


where $\mathfrak{S}_{p, \mathbb{C}}^{2}$ is the phase space $\mathfrak{S}_{p, \mathbb{C}}^{m}$ defined in (A.3) with $m=2$. We use the notation $i_{\xi} X_{P}$ for $X_{P}$ evaluated at $\xi$ and likewise in analogous cases. For each $\xi$, the vector field $i_{\xi} X_{P}$, considered as a map from a subset of $\mathfrak{S}_{p, \mathbb{C}}^{2}$ to $\mathfrak{S}_{p-2, \mathbb{C}}^{2}$, is of order $p-(p-2)=2$, which is strictly smaller than $d-1=4$. Moreover, it is easy to see that $i_{\xi} X_{P}$ is real analytic on $D(s, r)$ for each $\xi \in \Pi_{\varepsilon}$ and that $i_{w} X_{P}$ is uniformly Lipschitz on $\Pi_{\varepsilon}$ for each $w \in D(s, r)$. Namely, Assumption $C$ is satisfied.

Now we consider the supremum norm and Lipschitz seminorm of the perturbation $P$ on $D(s, r) \times \Pi$, where the parameter domain

$$
\Pi=\left\{\xi \in \mathbb{R}^{n}:|\xi| \leq r^{\frac{16}{\Pi}}\right\}
$$

Obviously, we have

$$
\left\|X_{Q}\right\|_{r, p-2, D(s, r) \times \Pi}=O\left(r^{2}\right) .
$$

Moreover, $\bar{P}$ is at least of the fifth order of $q$, so we get

$$
\left\|X_{\bar{P}}\right\|_{r, p-2, D(s, r) \times \Pi}=O\left(\left(r^{\frac{8}{11}}\right)^{5} \cdot r^{-2}\right)=O\left(r^{\frac{18}{11}}\right) .
$$

From (2.13) and (2.14) we have

$$
\left\|X_{P}\right\|_{r, p-2, D(s, r) \times \Pi}=O\left(r^{\frac{18}{11}}\right) .
$$

Since $X_{P}$ is real analytic in $\xi$, we have

$$
\left\|X_{P}\right\|_{r, p-2, D(s, r) \times \Pi}^{\operatorname{lip}}=O\left(r^{\frac{18}{11}} \cdot r^{-\frac{16}{11}}\right)=O\left(r^{\frac{2}{11}}\right) .
$$

We choose

$$
\alpha=r^{17} \gamma^{-1},
$$

where $\gamma$ is taken from KAM Theorem A.1. Set $M:=M_{1}+M_{2}$, which only depends on the index set $\mathcal{J}$. It is obvious that when $r$ is small enough,

$$
\left\|X_{P}\right\|_{r, p-2, D(s, r) \times \Pi}+\frac{\alpha}{M}\left\|X_{P}\right\|_{r, p-2, D(s, r) \times \Pi}^{\text {lip }}=O\left(r^{\frac{18}{11}}\right)=O(\varepsilon) \leq \alpha \gamma,
$$

which is just the smallness condition (A.5) in KAM Theorem A.1. Therefore Theorem 1.1 follows from Theorem A.1 in the Appendix.

\section{Conclusion}

Based on an abstract infinite dimensional KAM theorem for unbounded perturbation vector fields and partial Birkhoff normal form, we prove the existence of $n$-dimensional invariant tori for a nonlinearly modified Kawahara equation with periodic boundary conditions

$$
u_{t}+u_{x x x}-u_{5 x}+\alpha u^{2} u_{x}=0
$$




\section{Appendix: The KAM theorem}

Consider a small perturbation $H=N+P$ of an infinite-dimensional Hamiltonian in the parameter-dependent normal form

$$
N=\sum_{1 \leq j \leq m} \omega_{j}(\xi) y_{j}+\sum_{j \in \mathbb{N}_{*}} \Omega_{j} z_{j} \bar{z}_{j}
$$

on the phase space

$$
\mathfrak{S}_{p}^{m}=\mathbb{T}^{m} \times \mathbb{R}^{m} \times \ell^{p} \times \ell^{p} \ni(x, y, z, \bar{z})
$$

with symplectic structure

$$
\sum_{1 \leq j \leq m} \mathrm{~d} x_{j} \wedge \mathrm{d} y_{j}+\sum_{j \geq 1} \mathrm{~d} z_{j} \wedge \mathrm{d} \bar{z}_{j}
$$

where

$$
\ell^{p}=\left\{z \in \ell^{2}(\mathbb{N}, \mathbb{R}):\|z\|_{p}^{2}=\sum_{j \neq 0}\left|z_{j}\right|^{2} j^{2 p}<\infty\right\}, \quad p \geq 0 .
$$

The tangential frequencies $\omega=\left(\omega_{1}, \omega_{2}, \ldots, \omega_{m}\right)$ and normal frequencies $\Omega=\left(\Omega_{1}, \Omega_{2}, \ldots\right)$ are real analytic in the space coordinates and Lipschitz in the parameters. The Hamiltonian $N$ depends on parameters

$$
\xi \in \Pi \subset \mathbb{R}^{m},
$$

where $\Pi$ is a compact Cantor set in $\mathbb{R}^{m}$ of positive Lebesgue measure. Moreover, for each $\xi \in \Pi$, its Hamiltonian vector field

$$
X_{P}=\left(\left(P_{y_{b}}\right)_{1 \leq b \leq n},-\left(P_{\varphi_{b}}\right)_{1 \leq b \leq n}, \mathrm{i}\left(P_{\bar{z}_{j}}\right)_{j \in \mathcal{N}_{*}},-\mathrm{i}\left(P_{z_{j}}\right)_{j \in \mathcal{N}_{*}}\right)^{T}
$$

defines near $T_{0}:=\mathbb{T}^{m} \times\{y=0\} \times\{z=0\} \times\{\bar{z}=0\}$ a real analytic map

$$
X_{P}: \mathfrak{S}_{p}^{m} \rightarrow \mathfrak{S}_{q}^{m}
$$

where

$$
p-d=\tilde{d}
$$

To state the KAM theorem, we need to introduce some domains and norms. For $s, r>0$, we introduce the complex $T_{0}$-neighborhoods

$$
\begin{aligned}
D(s, r) & =\{|\Im \varphi|<s\} \times\left\{|y|<r^{2}\right\} \times\left\{\|z\|_{p}+\|\bar{z}\|_{p}<r\right\} \\
& \subset \mathbb{C}^{m} \times \mathbb{C}^{m} \times \ell_{\mathbb{C}}^{p} \times \ell_{\mathbb{C}}^{p}=\mathfrak{S}_{p, \mathbb{C}}^{m}
\end{aligned}
$$


and, for $W=(X, Y, Z, \bar{Z}) \in \mathfrak{S}_{q, \mathbb{C}}^{m}$, the weighted norm

$$
\|W\|_{r, q}=|X|+\frac{|Y|}{r^{2}}+\frac{\|Z\|_{q}}{r}+\frac{\|\bar{Z}\|_{q}}{r},
$$

where $|\cdot|$ denotes the sup-norm for complex vectors. Furthermore, for a map $W: U \times$ $\Pi \rightarrow \mathfrak{S}_{q, \mathbb{C}}^{m}$, such as the Hamiltonian vector field $X_{P}$, we define the norms

$$
\begin{aligned}
& \|W\|_{r, q ; U \times \Pi}^{\sup }=\sup _{(w, \xi) \in U \times \Pi}\|W(w, \xi)\|_{r, q}, \\
& \|W\|_{r, q ; U \times \Pi}^{\operatorname{lip}}=\sup _{\xi, \zeta \in \Pi, \xi \neq \zeta} \frac{\left\|\Delta_{\xi \zeta} W\right\|_{r, q ; U}^{\text {sup }}}{|\xi-\zeta|},
\end{aligned}
$$

where $\Delta_{\xi \zeta} W=i_{\xi} W-i_{\zeta} W$ and

$$
\left\|i_{\xi} W\right\|_{r, q ; U}^{\sup }=\sup _{w \in U}\|W(w, \xi)\|_{r, q}
$$

In a completely analogous manner, the Lipschitz seminorm of a frequency $\omega$ is defined as

$$
|\omega|_{\Pi}^{\operatorname{lip}}=\sup _{\xi, \zeta \in \Pi, \xi \neq \zeta} \frac{\left\|\Delta_{\xi \zeta} \omega\right\|}{|\xi-\zeta|}
$$

and the Lipschitz seminorm of $\tilde{\Omega}: \Pi \rightarrow \ell_{-\delta}^{\infty}$ is defined as

$$
|\tilde{\Omega}|_{-\delta, \Pi}^{\operatorname{lip}}=\sup _{\xi, \zeta \in \Pi, \xi \neq \zeta} \frac{\left\|\Delta_{\xi \zeta} \tilde{\Omega}\right\|_{-\delta}}{|\xi-\zeta|}
$$

for any real number $\delta$. Since $\bar{\Omega}=\Omega-\tilde{\Omega}$ is independent of $\xi$, we obtain $|\tilde{\Omega}|_{-\delta, \Pi}^{\text {lip }}=|\Omega|_{-\delta, \Pi}^{\text {lip }}$. For the normal form $N$ described previously, we introduce the following assumptions.

Assumption A (Frequency asymptotics) There exist two real numbers $d>1$ and $\delta<d-1$ such that the following condition holds. Firstly, the frequencies $\Omega_{n}$ are real-valued functions of $\xi$ of the form

$$
\Omega_{n}(\xi)=\bar{\Omega}_{n}+\tilde{\Omega}_{n}(\xi)
$$

where $\bar{\Omega}_{n}$ is independent of $\xi$ and the form $\tilde{\Omega}_{n}=c n^{d}+\cdots$, in which the dots stand for an expansion in lower order terms in $n$. Secondly, the functions

$$
\xi \mapsto \frac{\tilde{\Omega}_{n}(\xi)}{n^{\delta}}, \quad n \geq 1,
$$

are uniformly Lipschitz on $\Pi$, or, equivalently, the map

$$
\tilde{\Omega}: \Pi \rightarrow \ell_{-\delta}^{\infty}, \quad \xi \mapsto \tilde{\Omega}(\xi)=\left(\tilde{\Omega}_{n}(\xi)\right)_{n \geq 1}
$$

is Lipschitz on $\Pi$. 
Assumption B (Nondegeneracy) The map $\xi \rightarrow \omega(\xi)$ between $\Pi$ and its image is a homeomorphism, which is Lipschitz continuous in both directions. Moreover, for all $k \in \mathbb{Z}^{m}$ and $l \in \mathbb{Z}^{\infty}$ with $1 \leq|l| \leq 2$ (here $\left.|l|=\sum_{j \geq 1}\left|l_{j}\right|\right)$, the resonance set

$$
\Re_{k l}=\{\xi \in \Pi:\langle k, \omega(\xi)\rangle+\langle l, \Omega(\xi)\rangle=0\}
$$

has zero Lebesgue measure.

Assumption $\mathbf{C}$ (Regularity) There exists a neighborhood $U$ of $T_{0}$ in $\mathfrak{S}_{p, \mathbb{C}}^{m}$ such that $P$ is defined on $U \times \Pi$, and its Hamiltonian vector field defines a map

$$
X_{P}: U \times \Pi \rightarrow \mathfrak{S}_{q, \mathbb{C}}^{m},
$$

where $q$ satisfies

$$
p-q<d-1
$$

Moreover, $i_{\xi} X_{P}$ is real analytic on $U$ for each $\xi \in \Pi$, and $i_{w} X_{P}$ is uniformly Lipschitz on $\Pi$ for each $w \in U$.

We introduce one more constant. By Assumptions A and B,

$$
|\omega|_{\Pi}^{\operatorname{lip}}+|\Omega|_{\Pi}^{\operatorname{lip}} \leq M<\infty .
$$

Finally observe that if $X_{P}$ satisfies Assumption $C$, then it does so with the $T_{0}$-neighborhoods $D(s, r)$ for all $s>0$ and $r>0$ sufficiently small. Under the conditions stated, we have the following KAM theorem.

Theorem A.1 Suppose $N$ is a family of Hamiltonian of the form (A.1) defined on a phase space $\mathfrak{S}_{p}^{m}$ and depending on parameters in $\Pi$ so that Assumptions A and B are satisfied. Then there exist a positive constant $\gamma$, depending only on $m, d, \delta$, and the frequencies $\omega$ and $\Omega$, and the real number $s>0$ such that, for every perturbed Hamiltonian $H=N+P$ that satisfies Assumption $\mathrm{C}$ and the smallness condition

$$
\epsilon=\left\|X_{P}\right\|_{r, q, D(s, r) \times \Pi}^{\text {sup }}+\frac{\alpha}{M}\left\|X_{P}\right\|_{r, q, D(s, r) \times \Pi}^{\text {lip }} \leq \alpha \gamma
$$

for some $r>0$ and $0<\alpha<1$, the following holds: There exist

(i) a Cantor set $\Pi_{\alpha} \subset \Pi$ with meas $\left(\Pi \backslash \Pi_{\alpha}\right) \rightarrow 0(\alpha \rightarrow 0)$,

(ii) a Lipschitz family of real analytic torus embeddings $\Phi: \mathbb{T}^{m} \times \Pi_{\alpha} \rightarrow \mathfrak{S}_{p}^{m}$, and

(iii) a Lipschitz map $\phi: \Pi_{\alpha} \rightarrow \mathbb{R}^{m}$

such that, for each $\xi \in \Pi_{\alpha}$, the map $\Phi$ restricted to $\mathbb{T}^{m} \times\{\xi\}$ is a real analytic embedding of a rotational frequency $\phi(\xi)$ for the perturbed Hamiltonian $H$ at $\xi$. In other words,

$$
t \mapsto \Phi(\theta+t \phi(\xi), \xi), \quad t \in \mathbb{R}
$$

is a real analytic quasi-periodic solution for the Hamiltonian $i_{\xi} H$ for every $\theta \in \mathbb{T}^{m}$ and $\xi \in \Pi_{\alpha}$. 
Moreover, each embedding is real analytic on $D(s / 2)=\{|\mathfrak{s} x|<s / 2\}$, and

$$
\begin{aligned}
& \left\|\Phi-\Phi_{0}\right\|_{r, p, D(s / 2) \times \Pi_{\alpha}}^{\sup }+\frac{\alpha}{M}\left\|\Phi-\Phi_{0}\right\|_{r, p, D(s / 2) \times \Pi_{\alpha}}^{\operatorname{lip}} \leq \frac{c \epsilon}{\alpha}, \\
& |\phi-\omega|_{\Pi_{\alpha}}^{\sup _{\alpha}}+\frac{\alpha}{M}|\phi-\omega|_{\Pi_{\alpha}}^{\operatorname{lip}} \leq c \epsilon,
\end{aligned}
$$

where

$$
\Phi_{0}: \mathbb{T}^{m} \times \Pi \rightarrow T_{0}, \quad(x, \xi) \mapsto(x, 0,0,0)
$$

is the trivial embedding for each $\xi$, and $c$ is a positive constant depending on the same parameters as $\gamma$.

Proof The proof can be found in [16].

\section{Acknowledgements}

The authors would like to thank the editor and the anonymous referee for their valuable suggestions and comments, which help us to improve this paper greatly.

\section{Funding}

This work was supported by NSFC (Grant No. 11701320, 11601036) the Science and Technology Foundations of Shandong Province (Grant No. J16li52 and J17KA161) and Science Foundation of Binzhou University (Grant No. BZXYL1704)

\section{Availability of data and materials}

Not applicable.

\section{Competing interests}

The authors declare that they have no competing interests.

\section{Authors' contributions}

All authors contributed equally to the manuscript and read and approved the final manuscript.

\section{Author details}

${ }^{1}$ College of Science, Binzhou University, Binzhou City, China. ${ }^{2}$ College of Mathematics Science, Qufu Normal University, Qufu City, China.

\section{Publisher's Note}

Springer Nature remains neutral with regard to jurisdictional claims in published maps and institutional affiliations.

Received: 21 March 2019 Accepted: 24 June 2019 Published online: 04 July 2019

\section{References}

1. Abbasbandy, S.: Homotopy analysis method for the Kawahara equation. Nonlinear Anal., Real World Appl. 11(1), 307-312 (2010)

2. Baldi, P.: Periodic solutions of fully nonlinear autonomous equations of Benjamin-Ono type. Ann. Inst. Henri Poincaré Anal. Non Linéaire 30, 33-77 (2013)

3. Baldi, P., Berti, M., Montalto, R.: KAM for quasi-linear and fully nonlinear forced perturbations of Airy equation. Math. Ann. 359, 471-536 (2014)

4. Biswas, A.: Solitary wave solution for the generalized Kawahara equation. Appl. Math. Lett. 22, 208-210 (2009)

5. Bourgain, J.: Construction of quasi-periodic solutions for Hamiltonian perturbations of linear equations and applications to nonlinear PDE. Int. Math. Res. Not. 1994, 475-497 (1994)

6. Bourgain, J.: Construction of periodic solutions of nonlinear wave equations in higher dimension. Geom. Funct. Anal. 5, 629-639 (1995)

7. Bourgain, J.: Quasi-periodic solutions of Hamiltonian perturbations of $2 \mathrm{D}$ linear Schrödinger equations. Ann. Math. 148(2), 363-439 (1998)

8. Bourgain, J.: Green function estimates for lattice Schrödinger operators and applications. SIAM Rev. 47, 600-602 (2005)

9. Chierchia, L., You, J.-G.: KAM tori for 1D nonlinear wave equation with periodic boundary conditions. Commun. Math. Phys. 211, 498-525 (2000)

10. Craig, W., Wayne, C.E.: Newton's method and periodic solutions of nonlinear wave equations. Commun. Pure Appl. Math. 46, 1409-1498 (1993) 
11. Craig, W., Wayne, C.E.: Periodic solutions of nonlinear Schrödinger equations and the Nash-Moser method. In: Hamiltonian Mechanics, pp. 103-122. Springer, New York (1994)

12. Cui, W.-Y., Mi, L.-F., Yin, L.: Quasi-periodic solutions for non-autonomous MKdV equation. Indian J. Pure Appl. Math. 49(2), 313-337 (2018)

13. Cui, W.-Y., Mi, L.-F., Zhang, J.-M., Yin, L.: Invariant tori for a fifth order nonlinear partial differential equation. Dyn. Partial Differ. Equ. 15(3), 183-199 (2018)

14. Demina, M.V., Kudryashov, N.A.: From Laurent series to exact meromorphic solutions: the Kawahara equation. Phys. Lett. A 374(39), 4023-4029 (2010)

15. looss, G., Plotnikov, P., Toland, J.: Standing waves on an infinitely deep perfect fluid under gravity. Arch. Ration. Mech. Anal. 177, 363-478 (2005)

16. Kappeler, T., Pöschel, J.: KdV \& KAM. Springer, Berlin (2003)

17. Kawahara, T.: Oscillatory solitary. J. Phys. Soc. Jpn. 33(1), 260-264 (1972)

18. Kuksin, S.B.: Hamiltonian perturbations of infinite-dimensional linear systems with an imaginary spectrum. Funkc Anal. Prilozh. 21(3), 22-37 (1987)

19. Kuksin, S.B.: On small-denominators equations with large variable coefficients. Z. Angew. Math. Phys. 48, 262-271 (1997)

20. Kuksin, S.B.: On small denominators equations with large variable coefficients. Z. Angew. Math. Phys. 48, 262-271 (1997)

21. Kuksin, S.B.: Analysis of Hamiltonian PDEs. Oxford University Press, Oxford (2000)

22. Kuksin, S.B.: Fifteen years of KAM for PDE. In: Geometry, Topology, and Mathematical. American Mathematical Society Translations: Series 2, vol. 212, pp. 237-258 (2004)

23. Liu, J., Si, J.-G.: Invariant tori for a derivative nonliear Schrödinger equation with quasi-periodic forcing. J. Math. Phys. 56, 1-25 (2015)

24. Liu, J.-J., Yuan, X.-P.: Spectrum for equation Duffing oscillator and small-divisor equation with large-variable coefficient. Commun. Pure Appl. Math. 63, 1145-1172 (2010)

25. Liu, J.-.., Yuan, X.-P.: A KAM theorem for Hamiltonian partial differential equations with unbounded perturbations. Commun. Math. Phys. 307, 629-673 (2011)

26. Liu, J.-J., Yuan, X.-P.: KAM for the derivative nonlinear Schrödinger equation with periodic boundary conditions. J. Differ. Equ. 256, 1627-1652 (2014)

27. Marchenko, A.V.: Long waves in shallow liquid under ice cover. J. Appl. Math. Mech. 52(2), 180-183 (1988)

28. Mi, L.-F.: Quasi-periodic solutions of derivative nonlinear Schrödinger equations with a given potential. J. Math. Anal. Appl. 390, 335-354 (2012)

29. Mi, L.-F., Zhang, K.-K.: Invariant tori for Benjamin-Ono equation with unbounded quasi-periodically forced perturbation. Discrete Contin. Dyn. Syst. 34(2), 689-707 (2014)

30. Mi, L.-F., Zhang, K.-K.: Quasi-periodic solutions for perturbed generalized KdV equations. Nonlinear Anal., Real World Appl. 32, 314-337 (2016)

31. Natali, F:: A note on the stability for Kawahara-KdV equation. Appl. Math. Lett. 23, 591-596 (2010)

32. Si, J.-G.: Quasi-periodic solutions of a non-autonomous wave equations with quasi-periodic forcing. J. Differ. Equ. 197, 85-269 (2004)

33. Wang, Y:: Quasi-periodic solutions of a quasi-periodically forced nonlinear beam equations. Commun. Nonlinear Sci. Numer. Simul. 17, 2682-2700 (2012)

34. Wayne, C.E.: Priodic and quasi-periodic solutions of nonlinear wave equations via KAM theory. Commun. Math. Phys. $127,479-528(1990)$

35. You, J.-G.: Perturbations of lower dimensional tori for Hamiltonian system. J. Differ. Equ. 152, 1-29 (1999)

36. Yuan, X.P.: Construction of quasi-periodic breathers via KAM technique. Commun. Math. Phys. 226, 61-100 (2002)

37. Yuan, X.P.: Quasi-periodic solutions of nonlinear Schrödinger equations of higher dimension. J. Differ. Equ. 195, $230-242(2003)$

\section{Submit your manuscript to a SpringerOpen ${ }^{\circ}$ journal and benefit from:}

- Convenient online submission

- Rigorous peer review

- Open access: articles freely available online

- High visibility within the field

- Retaining the copyright to your article

Submit your next manuscript at $\gg$ springeropen.com 